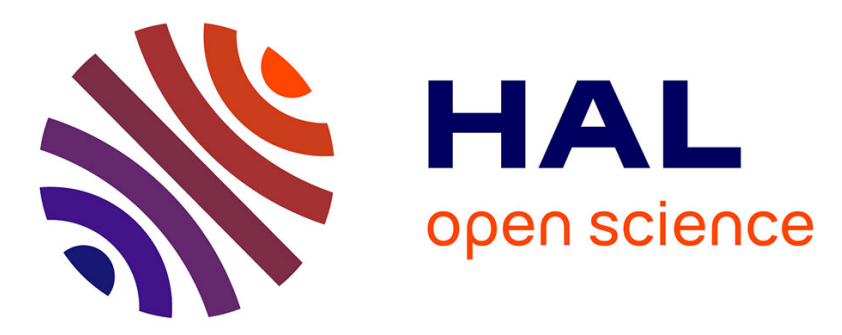

\title{
Ressource en eau et fabrique du risque sanitaire au prisme de l'analyse de la vulnérabilité dans la capitale malgache
}

Carole Ognard, François Taglioni

\section{- To cite this version:}

Carole Ognard, François Taglioni. Ressource en eau et fabrique du risque sanitaire au prisme de l'analyse de la vulnérabilité dans la capitale malgache. Les Cahiers d'Outre-Mer. Revue de géographie de Bordeaux, 2019, 280, pp.339-367. 10.4000/com.10528 . hal-02909699

\section{HAL Id: hal-02909699 \\ https://hal.science/hal-02909699}

Submitted on 1 Aug 2020

HAL is a multi-disciplinary open access archive for the deposit and dissemination of scientific research documents, whether they are published or not. The documents may come from teaching and research institutions in France or abroad, or from public or private research centers.
L'archive ouverte pluridisciplinaire HAL, est destinée au dépôt et à la diffusion de documents scientifiques de niveau recherche, publiés ou non, émanant des établissements d'enseignement et de recherche français ou étrangers, des laboratoires publics ou privés. 


\title{
RESSOURCE EN EAU ET FABRIQUE DU RISQUE SANITAIRE AU PRISME DE L'ANALYSE DE LA VULNÉRABILITÉ DANS LA CAPITALE MALGACHE
}

\author{
Carole Ognard, François Taglioni
}

Presses universitaires de Bordeaux | «Les Cahiers d'Outre-Mer »

2019/2 n 280 | pages 439 à 467

ISSN 0373-5834

ISBN 9791030005783

Article disponible en ligne à l'adresse :

https://www.cairn.info/revue-les-cahiers-d-outre-mer-2019-2-page-439.htm

Distribution électronique Cairn.info pour Presses universitaires de Bordeaux.

(C) Presses universitaires de Bordeaux. Tous droits réservés pour tous pays.

La reproduction ou représentation de cet article, notamment par photocopie, n'est autorisée que dans les limites des conditions générales d'utilisation du site ou, le cas échéant, des conditions générales de la licence souscrite par votre établissement. Toute autre reproduction ou représentation, en tout ou partie, sous quelque forme et de quelque manière que ce soit, est interdite sauf accord préalable et écrit de l'éditeur, en dehors des cas prévus par la législation en vigueur en France. Il est précisé que son stockage dans une base de données est également interdit. 


\section{Les Cahiers d'Outre-Mer}

Revue de géographie de Bordeaux

\section{Ressource en eau et fabrique du risque sanitaire au prisme de l'analyse de la vulnérabilité dans la capitale malgache}

\section{Carole Ognard et François Taglioni}

\section{(2) OpenEdition Journals}

Édition électronique

URL : http://journals.openedition.org/com/10528

DOI : $10.4000 /$ com. 10528

ISSN : 1961-8603

Éditeur

Presses universitaires de Bordeaux

Édition imprimée

Date de publication : 1 juillet 2019

Pagination : 439-467

ISBN : 979-10-300-0578-3

ISSN : 0373-5834

Distribution électronique Cairn

\section{CAIRN INFC}

CHERCHER, REPÉRER, AVANCER.

\section{Référence électronique}

Carole Ognard et François Taglioni, « Ressource en eau et fabrique du risque sanitaire au prisme de I'analyse de la vulnérabilité dans la capitale malgache », Les Cahiers d'Outre-Mer [En ligne], 280 | JuilletDécembre, mis en ligne le 02 janvier 2024, consulté le 27 juillet 2020. URL : http:// journals.openedition.org/com/10528; DOI : https://doi.org/10.4000/com.10528 


\title{
Ressource en eau et fabrique du risque sanitaire au prisme de l'analyse de la vulnérabilité dans la capitale malgache
}

\author{
Carole Ognard ${ }^{1}$ et François Taglioni ${ }^{2}$
}

\section{Résumé}

Cette étude démontre que la vulnérabilité de la ressource en eau dépend d'un système en chaîne de la « source à la consommation » où toutes les interventions peuvent détériorer l'accès à l'eau potable en quantité et en qualité, en particulier dans les quartiers aux habitats précaires et denses. À Antananarivo, seuls $33 \%$ de la population ont un accès à un branchement individuel, ce qui oblige le reste de la population à recourir à des pratiques de transport et de stockage à partir des bornesfontaines, parfois aux dépens de leur état de santé. Cette dépendance du réseau à un fonctionnement en système d'amont en aval, est ici qualifiée de «vulnérabilité linéaire » de l'approvisionnement en eau. Parallèlement, la rivière Ikopa à la fois source et effluent, participe à la « vulnérabilité transversale » de la capitale car les infrastructures critiques de l'eau et de l'assainissement interagissent et influencent l'état de santé de la population. Ce bien vital qu'est la ressource en eau y acquiert ainsi le statut ambivalent de ressource menacée et menaçante et participe d'une fragmentation socio-spatiale de la capitale malgache.

Mots-clés : eau potable, vulnérabilité, qualité de l'eau, santé, fragmentation socio-spatiale.

\section{Abstract}

This study shows that the vulnerability of water resources depends on a chain system from «source to consumption», where all interventions can deteriorate access to drinking water in quantity and quality, particularly in neighbourhoods with precarious and dense settlements. In Antananarivo, only $33 \%$ of the population has access to 
an individual connection, forcing the rest of the population to resort to transport and storage practices from standpipes, sometimes at the expense of their health. The network's dependence on an upstream-downstream system is referred to here as «linear vulnerability» of the water supply. At the same time, Ikopa River, which is both source and effluent, contributes to the "transversal vulnerability» of the capital because the critical water and sanitation infrastructures interact and influence the health status of the population. This vital asset of water resources thus acquires the ambivalent status of a threatened and endangered resource and contributes to the socio-spatial fragmentation of the Malagasy capital.

Keywords : Drinking water, vulnerability, water quality, health, socio-spatial fragmentation.

\section{Introduction}

Dans la Commune urbaine d'Antananarivo ${ }^{3}$ (CUA) (figure 1), seuls $33 \%$ de la population bénéficient d'un branchement individuel à l'eau (Banque mondiale, 2011). Dans un contexte de croissance démographique d'une capitale devenue bi-millionaire, les graves lacunes sur le plan de la distribution et de la régulation créent des déséquilibres accrus entre l'offre et la demande en particulier pour les populations les plus démunies comme celles de la plaine à l'ouest de la CUA (figure 1). Cette répartition inégale des infrastructures et de la quantité d'eau trouve son corollaire avec le développement de maladies hydriques comme la résurgence du choléra (Duston et Mac Afee, 2001). Plus communément, les maladies diarrhéiques qui figurent parmi les deux premières causes de consultation des Centres de santé de base publics (CSB) de la capitale, en particulier chez les enfants, constituent un véritable problème de santé publique (Ministère de la santé, 2007-2012). En réalité, le danger sur la ressource en eau ne se limite pas à la seule eau réputée « potable » à son lieu de distribution. Car comme dans la plupart des pays du Sud (Sy et Moussa, 2014), le paysage des bornes-fontaines domine à Antananarivo. De fait, la vulnérabilité de la ressource intègre également les questions de transport et de stockage, et notamment celle des manipulations de l'eau dans la sphère domestique qui peuvent contaminer les eaux et endommager la santé (Dos Santos, 2005 ; Lavie et Hassan El-Tayib, 2014 ; Amrose et al., 2015). L'accès à la ressource en eau peut être ici analysé comme un indicateur de vulnérabilité urbaine antananarivienne puisqu'elle est une des composantes essentielles et un bien de l'environnement urbain (D'Ercole et Metzger, 2008 ; Reghezza et Ruffat, 2015). Ce bien vital qu'est l'eau domestiquée acquiert un statut ambivalent de ressource menacée et menaçante. C'est une problématique complexe liant santé et environnement urbain. L'étude de la vulnérabilité territoriale de la capitale malgache vis-à-vis de la ressource en eau est ici 


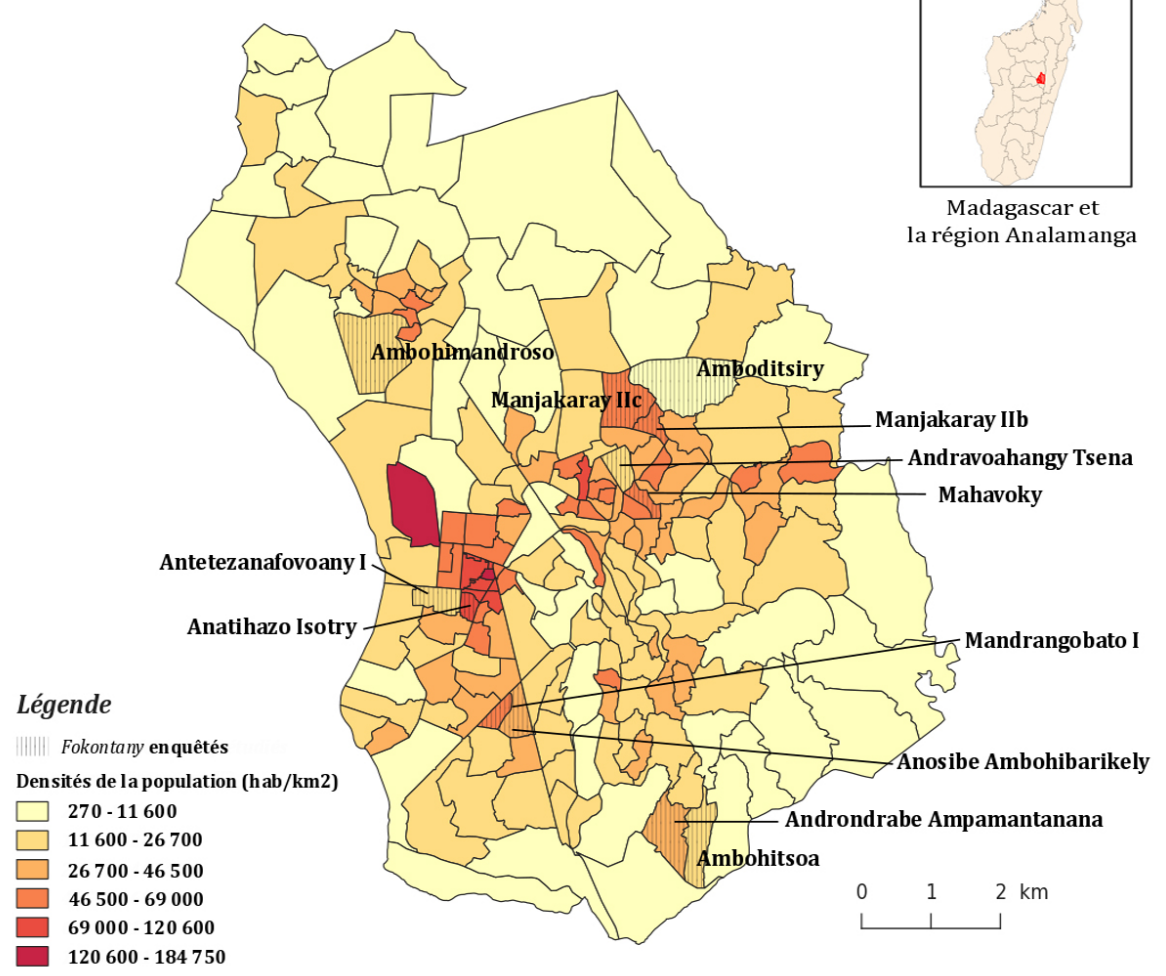

Figure 1 - Carte de localisation de la Commune Urbaine d'Antananarivo et des fokontany enquêtés

Source: ARTELIA, 2014. Réalisation : Ognard, 2018

illustrée au travers d'espaces fragilisés, à savoir les quartiers qui présentent des densités fortes et aux formes d'habitats précaires et dans lesquels se trouvent les personnes qui comptent le moins dans les représentations sociales et où se trouvent les besoins de recherche (figure 1). En plaçant ces écosystèmes fragilisés au cœur de l'analyse à travers le prisme des concepts de risque et vulnérabilité, notre hypothèse est que l'eau salubre de la source à la consommation devrait être considérée comme un système en chaîne (Burt et Ray, 2014 ; Amrose et al., 2015). Ainsi, nous cherchons à déterminer toutes les interventions qui pourraient détériorer l'accès à l'eau potable en quantité et en qualité dans ces quartiers de la capitale. Cela revient à approcher la question de la sécurité de l'approvisionnement en eau en quantité et en qualité.

L'analyse commence par dresser un tableau de la thématique de la pénurie de l'eau dans les villes du Sud et de sa réalité dans la capitale malgache. Nous 
soutenons ensuite qu'une approche holistique est nécessaire pour aborder les domaines multiples qui influencent l'état de santé dans un contexte d'environnement urbain où la carence en services urbains comme l'accès à l'eau est évidente.

\section{I- Le système d'eau potable dans les pays du Sud: de la source à la consommation}

L'eau potable salubre est essentielle à une vie saine et digne et a été reconnue comme un droit par l'ONU (ONU, 2010). Pourtant dans les pays du Sud, beaucoup n'ont pas accès à l'eau courante, et parmi ceux qui y ont accès, beaucoup reçoivent une eau de qualité douteuse. Il existe de nombreux contaminants biologiques et chimiques dans l'eau potable et nous limitons la portée de cet article à la contamination physique et microbienne. La contamination microbienne est de loin le plus grand danger pour l'eau potable dans les pays du Sud. Au moins 1,8 milliard de personnes n'ont pas accès à une eau potable fiable (Onda et al., 2012). Près de 1000 décès d'enfants par jour résultent de maladies diarrhéiques causées par une eau insalubre, un assainissement insuffisant et une mauvaise hygiène (OMS, 2014 ; Taglioni, 2019). Les systèmes d'eau salubre de la source à la consommation ont été conceptualisés comme une série d'étapes comprenant les technologies de traitement, les modèles de distribution et les pratiques de transport et de stockage avant consommation car ils déterminent conjointement la salubrité de l'eau avant sa consommation (Amrose et al., 2015). Or, dans la littérature, la sécurité de l'approvisionnement en quantité et en qualité est souvent traitée séparément. On retrouve des articles focalisés sur les réseaux à travers les questions d'accès en quantité alors que les approches par les ménages s'intéressent à la qualité de l'eau et l'impact sur la santé.

\section{1- Les approches par le réseau centralisé}

L'OMS considère l'accès à l'eau courante dans le logement, la parcelle ou la cour comme la forme d'accès la plus améliorée. Au niveau mondial, $56 \%$ de la population avaient accès à l'eau courante en 2012 (OMS, 2014). Outre l'accès à l'eau courante, les bornes-fontaines publiques, les puits et les sources d'eau protégées sont également classés comme améliorés. Au niveau mondial, $33 \%$ de la population y avaient accès en 2012 (OMS, 2014). L'abondante littérature sur l'efficacité des services publics de distribution d'eau dans les pays du Sud est principalement axée sur les volumes livrés. Car si l'évaluation de la sécurité de l'approvisionnement de la ressource en eau pose d'abord celle de sa disponibilité (Falkenmark, 1989 ; Bravard et Honnegger, 2006 ; Jemmali, 
2013), la crise de l'eau dans les villes du Sud n'est pas moins un problème de pénurie qu'une «pénurie infrastructurelle » (Lavie et Hassan El-Tayib, 2014 ; Lavie et al., 2015). En effet, la plupart des citadins ne sont pas desservis par des systèmes d'approvisionnement en eau courante, mais la réalité est loin d'y être uniforme (Bromley, 1978). Elle se présente comme un modèle hybride de stratégies superposées d'approvisionnement (Bakker, 2003). Loin d'une vision simpliste duale entre modes d'approvisionnement artisanaux et modes industriels, Bakker argumente que cet « archipel » de services formel et informel coexiste parce que le système formel est spatialement inégal (Bakker, 2003). En effet, là où le niveau des infrastructures est déficient, les modes d'approvisionnement informels dominent. Or, à l'origine ces services urbains ont été théorisés comme des moyens d'assurer l'équité sociale et territoriale car de tels systèmes sont couramment considérés comme des préalables à la vie urbaine moderne. Jaglin a ainsi identifié l'échec de ce modèle universaliste d'expansion des services avec la remise en cause des monopoles publics tout autant que des expériences récentes de partenariat public-privé (Jaglin, 2005). Plusieurs auteurs s' accordent pour affirmer que la nature du problème est avant tout politique (Jaglin, 2005 ; Blanchon, 2010 ; Aubriot et Riaux, 2013 ; Burt et Ray, 2014 ; Ginisty, 2014 ; Lavie et al., 2015). Ces défaillances de régulation et de gouvernance sont le reflet de la « dépolitisation des rapports de force des sociétés » (Jaglin et Zérah, 2010). «La propension à la réalisation du modèle universaliste relève avant tout de la capacité à construire l'intérêt national et de l'inclusion dans les sociétés fragmentées », ce qui s'avère difficile à mettre en œuvre dans des contextes fragilisés des pays du Sud. L'informalité urbaine a été conceptualisée comme " un mode de vie urbain » et " un ensemble de stratégies de survie par les pauvres» (De Soto, 2000) et une «forme de résistance aux élites urbaines » (Bayat, 2000). L'analyse sous le prisme du concept de risque semble ici convenir. La vulnérabilité n'est pas seulement une propension à subir les dommages mais intègre aussi une capacité à les provoquer, les modifier (Reghezza-Zitt et Ruffat, 2015). En fonction de l'estimation du danger et des priorités d'action, certains risques deviendront matérialisés et de là, politisés ; au contraire de certains autres qui resteront oubliés, parfois intentionnellement. Chaque groupe humain, en définissant un ordre social, lui-même producteur de hiérarchie, donne à voir le choix des risques à craindre, des enjeux qui n'apparaissent comme « ce que l'on est prêt à perdre » (Metzgler et D’Ercole, 2009).

\section{2- Les approches axées sur les ménages}

Aussi, tout ménage sans accès à l'eau courante doit transporter et stocker son eau potable. Si l'eau est salubre au point d'accès, le stockage sécuritaire 
peut fournir une certaine protection contre la contamination à la maison. On entre alors dans la question des pratiques. Malgré une bibliographie pourtant fournie sur la gestion, les pratiques et l'accès à l'eau dans les villes africaines (Dos Santos, 2005 ; Jaglin, 2005 ; Blanchon, 2010 ; Burt et Ray, 2014 ; Ginisty, 2014), il est rare de disposer de référence sur les qualités physique, chimique et biologique de cette eau consommée (Amrose et al., 2015). Elles prennent souvent la forme d'un suivi hydro-chimique. Les rares exceptions de prise en compte de façon conjointe du suivi hydro-chimique, des pratiques sociales et de gestion concernent les travaux de Nguimalet et al. (2005) menant une étude systémique mêlant diagnostic hydro-qualitatif et analyse des gestions de l'eau potable dans la ville de Bangui en République centrafricaine et les travaux d'Emilie Lavie et de Noha Hassan El-Tayib, s'intéressant aux pratiques de stockage qui sont responsables d'une baisse significative de la qualité physique et biologique de l'eau dans la ville de Khartoum et d'une incidence sur les maladies hydriques (Lavie et Hassan El-Tayib, 2014).

\section{3- L'approche par le « système d'eau potable : de la source à la consommation »}

Pour intégrer ces deux dimensions de la quantité et de la qualité, nous empruntons ici au modèle conceptuel «from source to sip » (Amrose et al., 2015). Tous les systèmes d'eau potable salubre comportent cinq étapes qui commencent à la source d'eau et se terminent au point de consommation : la source, le transport et stockage à la source, un point d'accès public ou privé, le transport et stockage (et parfois traitement) au-delà du point d'accès, et la consommation. Le traitement avant l'accès doit être mis en œuvre par les services publics. Le transport de l'eau s'effectue dans les pays du Sud d'abord par des canalisations puis à partir des points d'eau collectifs à l'aide de seaux, barils, charrettes et camions. Après la collecte, un traitement peut être effectué par le ménage. En suivant le trajet de l'eau depuis le captage jusqu'au ménage, toutes les étapes déterminent l'efficacité du système. L'organisation des réseaux ne se réduit pas seulement à celle d'un système technique mais interfère dans l'organisation sociale et spatiale de la ville. Ils sont à la fois vulnérables aux risques et propagateurs de perturbations, à cause de leurs interdépendances (Hardy, 2009 ; Reghezza-Zitt, Rufat, 2015). La vulnérabilité renvoie à l'idée qu'il existe au sein de tout territoire des éléments localisables susceptibles d'engendrer ou de diffuser leur vulnérabilité sur l'ensemble de leur territoire, provoquant des effets qui peuvent perturber, compromettre voire interrompre son fonctionnement (Metzger et D'Ercole, 2011). Dans cette logique, l'analyse de vulnérabilité territoriale vise prioritairement à identifier, caractériser et hiérarchiser les espaces à partir desquels se crée et se diffuse la vulnérabilité au sein du territoire. Il existe 
Domicile équipé

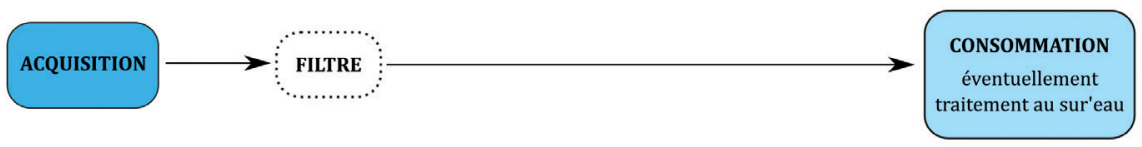

Domicile non équipé - Approvisionnement Bornes Fontaines

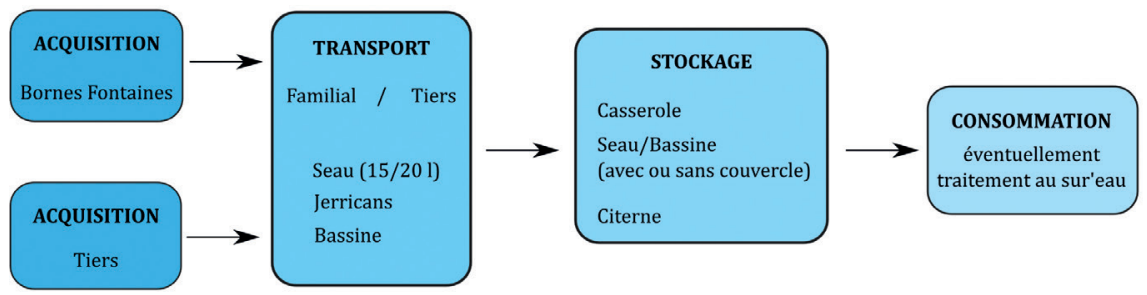

Domicile non équipé - Source non protégée

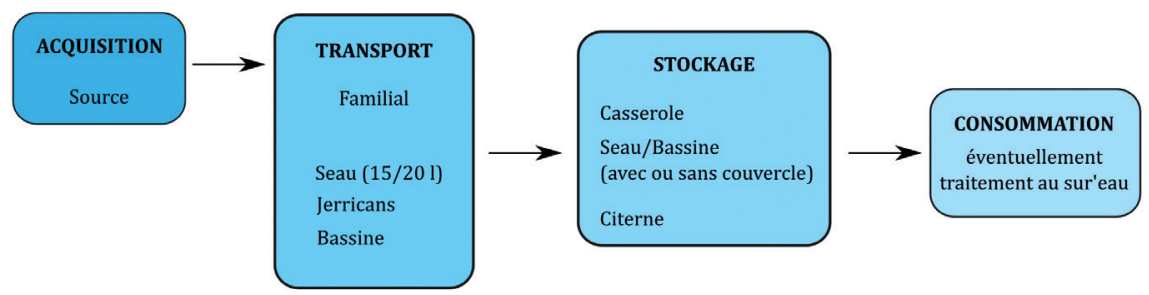

Figure 2 - Le système de l'eau antananarivien :

de la source à la consommation

Source : D'après Lavie, Hassan, 2014. Réalisation : Ognard, 2018.

deux types de vulnérabilité de ces approvisionnements (Hardy, 2009). Elle est d'abord linéaire. En effet, les réseaux d'eau et d'assainissement dépendent d'un fonctionnement en système d'amont en aval. Une perturbation sur l'un des maillons peut engendrer une crise sur l'ensemble du système. Parallèlement, la vulnérabilité des approvisionnements est transversale car la plupart de ces infrastructures interagissent. La perte d'approvisionnement ou d'assainissement peut provoquer d'autres problèmes de fonctionnement $\mathrm{du}$ système urbain. Les réseaux techniques et critiques font figure d'éléments stratégiques de la résilience urbaine. En effet, en évaluant la capacité des réseaux à fonctionner en mode dégradé, on peut identifier les dysfonctionnements possibles et la diffusion des effets délétères. Samuel Rufat définit les bidonvilles comme les territoires les plus proches de la définition de la ville résiliente car ils sont capables « de fonctionner en état dégradé » ou car ils facilitent « une reconstruction rapide» (Ruffat, 2015). Pour lui, ce sont dans ces territoires que se retrouvent le plus souvent les catastrophes. Loin des discours enthousiastes, cette « mauvaise résilience est passée sous 


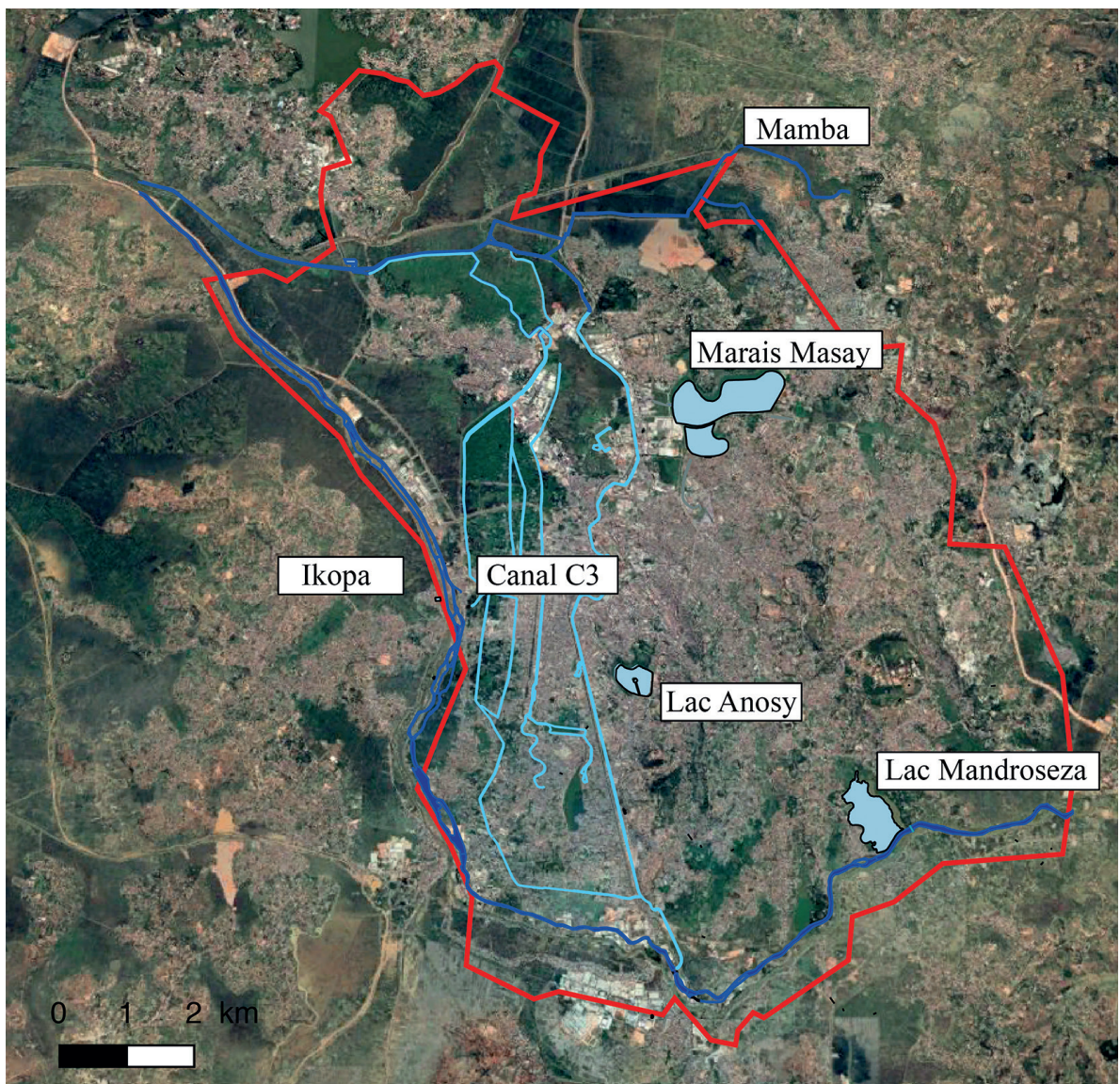

Figure 3 - Carte de localisation du réseau d'adduction d'eau potable de la Commune urbaine d'Antananarivo

Source : D'après Lavie, Hassan, 2014. Réalisation : Ognard, 2018.

silence mais constitue l'ordinaire des catastrophes urbaines. Elle est comme invisible car c'est le quotidien des habitants du côté de la ville ordinaire et du bricolage face à l'adversité plus que du côté spectaculaire des crises et des catastrophes » (Rufat, 2015). Ainsi les quartiers informels ou bidonvilles à l'écart des réseaux de viabilité sont intrinsèquement la structure qui peut le plus fonctionner en état dégradé. Dans la littérature anglo-saxonne, on retrouve cette idée à travers le modèle de Bayat qui, dépassant les dissensions entre les «passive coying » et l' "active resistance » paradigme, affirme que les services informels sont ceux du « silencieux empiétement de l'ordinaire » par lequel les populations pauvres sans bruit et sans fureur outrepassent les canaux ordinaires des services urbains (Bayat, 2000 ; De Soto, 2000). 


\section{4- Le système de l'eau antananarivien : de la source à la consommation}

Dans la capitale malgache, les habitants accèdent à la ressource suivant deux modalités : le réseau d'approvisionnement par canalisation qui alimente à la fois les particuliers et les bornes-fontaines essentiellement publiques de la ville et les rares puits observés (figure 2). Or, la capitale est alimentée en eau à partir d'un site de production unique, situé à Mandroseza, au sudest de la ville, en bordure nord-ouest du lac appelé également Mandroseza (figure 3). Ce lac, naturel à l'origine, est alimenté par la rivière Ikopa qui contourne la ville par le sud. La construction de la voie ferrée de Tana-Côte Est en 1909 avait mis en place la digue en rive droite de l'Ikopa. Cette digue forme la rétention de l'eau sur le lieu marécageux. Et le remplissage d'eau de l'espace forme le lac Mandroseza qui a une superficie de 47 ha environ pour une profondeur moyenne de 2,5 $\mathrm{m}$ et assurant un volume de stockage de 1,2 million de $\mathrm{m}^{3}$ (Chardon, 1996). C'est donc sur ce site que sont concentrées les installations de Mandroseza I et Mandroseza II. Construites au fur et à mesure de l'accroissement des besoins de la population, les premières installations de production datant du début du siècle et la plus récente mise en service en 1993, attestent de la vétusté des infrastructures (Chardon, 1996). Au total, le site de Mandroseza pourrait donc produire au maximum de ses capacités, près de $150000 \mathrm{~m}^{3}$ d'eau traitée par jour. Compte tenu de la vétusté du système de production, de refoulement et de distribution de l'eau potable, le dispositif distribue $140500 \mathrm{~m}^{3}$ par jour et n' alimente que $33 \%$ de la population de la CUA et $14 \%$ de la périphérie en branchement individuel (ARTELIA, 2014). Pour fonctionner, un système a besoin des maillons précédents, chaque élément du système dépend de celui situé en amont. Aussi, en termes de sécurité d'approvisionnement, la capitale apparaît comme vulnérable en considération de l'unique site d'approvisionnement de la ville. À ce titre, une simulation d'un risque de pollution a été potentiellement envisagée par la CUA (CUA, 2014) selon deux scénarios distincts : celui du déraillement d'un train transportant des produits chimiques ou du fuel, ou un empoisonnement volontaire à Mandroseza. En cas de contamination du lac Mandroseza, l'eau stockée dans les 37 réservoirs d'eau de la ville ne pourra assurer avec ses $150000 \mathrm{~m}^{3}$, qu'un jour d'approvisionnement. Si improbable soit l'occurrence de tels événements, cette simulation marque cependant une conscientisation de la «vulnérabilité linéaire » de cet approvisionnement, c'est-à-dire d'un fonctionnement en amont-aval.

À l'échelle intra-urbaine, une évaluation de l'accès à l'eau potable a été menée dans le cadre de la réalisation du Schéma d'aménagement urbain (SDAU) 
pour les 24 communes couvertes par le réseau de distribution de la JIRAMA ${ }^{4}$, représentant près de $90 \%$ de la population totale de la zone d'étude (ARTELIA, 2014). Au regard de la croissance assez rapide de la population au sein de l'agglomération, la JIRAMA fait face à d'énormes contraintes pour répondre à la demande toujours grandissante, en termes de services urbains d'accès à l'eau potable, qui est identifiable à travers le taux de desserte. Ce taux traduit la part de la population qui a accès à l'eau potable de manière permanente par les branchements particuliers et les bornes-fontaines sur l'effectif total de la population, et ce pendant une année donnée. D'après les données du SDAU, la situation de l'approvisionnement en eau potable s'est beaucoup dégradée depuis l'année $2002^{5}$. Entre 2002 et 2012, le taux d'accès à l'eau potable a considérablement régressé d'au moins $31 \%$ (de $65 \%$ à $45 \%$ ). Le nombre de bénéficiaires sur l'ensemble de l'agglomération ne s'élevant qu'à environ un million d'habitants. Une part importante résidant dans les zones péri-urbaines n'a pas encore accès de manière permanente à l'eau potable. Il apparaît que certaines communes sont même dépourvues d'infrastructures. La Commune urbaine d'Antananarivo apparaît la mieux lotie avec un taux de desserte de $47 \%$. En revanche, une situation très disparate de la desserte en eau entre les arrondissements est à signaler pour la CUA (tableau 1). De fait, la population s'approvisionne pour une majeure partie aux bornes-fontaines et la question du transport et stockage est donc prépondérante (figure 2).

Tableau 1 - Situation de I'approvisionnement en eau en 2012

\begin{tabular}{|c|c|c|c|c|c|}
\hline \multirow{2}{*}{$\begin{array}{l}\text { Arrondissements } \\
\text { et communes }\end{array}$} & \multicolumn{3}{|c|}{ Taux de desserte (\%) } & \multicolumn{2}{|c|}{ Dotation unitaire ( $1 / \mathrm{j} /$ pers) } \\
\hline & Global & $\begin{array}{c}\text { Branchement } \\
\text { Particulier }\end{array}$ & $\begin{array}{l}\text { Borne- } \\
\text { fontaine }\end{array}$ & $\begin{array}{c}\text { Branchement } \\
\text { Particulier }\end{array}$ & Borne-Fontaine \\
\hline $1^{\mathrm{er}}$ arrondissement & $51 \%$ & $61 \%$ & $39 \%$ & 113 & 37 \\
\hline $2^{\mathrm{e}}$ arrondissement & $52 \%$ & $52 \%$ & $48 \%$ & 112 & 27 \\
\hline $3^{\mathrm{e}}$ arrondissement & $75 \%$ & $66 \%$ & $34 \%$ & 115 & 25 \\
\hline $4^{\mathrm{e}}$ arrondissement & $37 \%$ & $36 \%$ & $64 \%$ & 153 & 30 \\
\hline $5^{\mathrm{e}}$ arrondissement & $42 \%$ & $58 \%$ & $42 \%$ & 111 & 30 \\
\hline $6^{\mathrm{e}}$ arrondissement & $30 \%$ & $44 \%$ & $57 \%$ & 106 & 41 \\
\hline Moyenne CUA & $48 \%$ & $53 \%$ & $47 \%$ & 118 & 32 \\
\hline
\end{tabular}

Source : ARTELIA, 2014.

4. Acronyme malgache pour « jiro sy rano malagasy », soit eau et électricité de Madagascar.

5. Année de référence pour l'élaboration du schéma directeur d'alimentation en eau potable de la ville d'Antananarivo. 


\section{II- Un sujet d'interface qui requiert une démarche à la méthodologie fondée sur une approche pluridimensionnelle}

\section{1- Démarche pluridimensionnelle et approche intégrée en environnement et santé : les maladies hydro-dépendantes}

L'eau imprègne une multiplicité des domaines du réel (Casciarri et Van Aken, 2013). Contrairement à la plupart des risques sanitaires, les risques hydriques développent une originalité compte tenu de leur autre dimension, la dimension environnementale. Objets complexes à l'interface santél environnement, ils interrogent la méthodologie et présente une vocation pluridimensionnelle. Nous soutenons qu'une approche pluridimensionnelle soulignant les interdépendances entre le politique, le social, le technique et la contamination microbiologique de l'eau potable, peut nous aider à comprendre à la fois la répartition inégale de la quantité et de la qualité de l'eau (Aubriot et Riaux, 2013 ; Sy et Moussa, 2014 ; Amrose et al., 2015). Ceci est d'autant plus vrai que l'on se trouve dans un territoire qui connaît des crises itératives et multiformes et où l'accès aux données est difficile (Fremigacci, 2014 ; Razafindrakoto et al., 2014 ; Roubaud, 2017). Sur le plan méthodologique, il s'agit de combiner des approches quantitatives et qualitatives avec des méthodes permettant de quantifier les flux d'eau matérielle et d'évaluer la contamination microbiologique (Rusca et al., 2017). Or la géographie comme discipline de synthèse des sciences connexes, trouve ici à l'interface environnement-santé et particulièrement dans le cas des risques infectieux hydriques, un domaine d'application fécond. Cette démarche se veut holistique pour aborder les domaines multiples qui influencent l'état de santé.

\section{2- Description du site}

Antananarivo est située dans les hauts plateaux du centre de Madagascar à $1300 \mathrm{~m}$ d'altitude. La ville est nichée au milieu de 12 collines et se localise sur la plaine d'inondation naturelle de la rivière Ikopa, qui longe la ville au sud et à l'ouest. L'aire métropolitaine s'étend sur $220 \mathrm{~km}^{2}$ avec une population estimée à 1,5 million d'habitants. L'unité administrative sur laquelle se focalise cette recherche est celle des fokontany, unité administrative de base à Madagascar, significativement calquée sur le modèle-villageois (Fournet-Guérin, 2006) ; Antananarivo en compte 192. Dans une démarche comparative deux fokontany par arrondissements ont été sélectionnés, la CUA en comptant 6 , soit 12 fokontany (figure 1). 


\section{1- Phase exploratoire et préparatoire au terrain}

Il est très vite apparu dans le cadre de cette recherche que le territoire antananarivien peut être qualifié de " rugueux » au vu des nombreuses difficultés rencontrées. Tout d'abord, face au climat de crise multiforme et itérative qui caractérise Madagascar et Antananarivo, l'accès au terrain a dû constamment être négocié c'est-à-dire toujours contraint par plusieurs entraves administratives et de fait les résultats ne sont pas toujours aussi fructueux qu'attendus. En effet, la structure verticale du pouvoir, propre à ce type de territoire fait qu'il est à chaque fois indispensable d'avoir l'aval du responsable de niveau supérieur pour réaliser enquêtes et entretiens. Depuis son indépendance, Madagascar affiche une trajectoire économique en récession continue. Au final, ces crises s'enchaînent pour ne former qu' une seule et même crise structurelle de l'État postcolonial (Fremigacci, 2014). Cette « énigme » de la cyclicité des crises et le « paradoxe » de leur imbrication entre croissance et politique montrent un décalage croissant entre élites et populations locales (Razafindrakoto et al., 2014 ; Roubaud, 2017). De fait, la recherche en territoire malgache et antananarivien se heurte à la qualité des données au vu des statistiques disponibles biaisées par l'ancienneté du recensement. Celui-ci date de 1993 et les données alors obtenues ne correspondent plus à la réalité. Les données de recensement diffusées par l'Institut national de la statistique (INSTAT) depuis 2003 sont pour la plupart extrapolées avec un taux de croissance de $2,5 \%$. Face à ce manque de fiabilité des données statistiques (Razafindrakoto et al., 2014 ; Roubaud, 2017), il nous est apparu essentiel d'élaborer nos propres enquêtes sous diverses dimensions pour approcher au plus près la réalité locale, soulignant ainsi l'importance et la centralité du travail de terrain. D'ailleurs, cette réalité locale s'est rapidement heurtée à notre cadre de référencement occidental et à ses repères. Par exemple, dans un même habitat peuvent coexister plusieurs chefs de ménages et donc plusieurs ménages (plus de $44 \%$ des ménages sont composés de 5 individus et plus des $2 / 3$ composés de plus de 2 enfants, chaque ménage possède ou loue dans la maison une à deux pièces : les équipements de cuisine et sanitaire étant au mieux communautaire et au pire inexistant).

\section{2- Collecte de données quantitatives et qualitatives}

Cette recherche est issue d'un doctorat mené entre 2011 et 2018, avec cinq missions réparties selon le tableau suivant (tableau 2). Les 4 dernières missions avaient pour objectif la réalisation de différentes enquêtes permettant d'embrasser les diverses questions de ce sujet d'interface, d'où le caractère composite des données récoltées. Elles sont à la fois quantitatives et qualitatives. 
Tableau 2 - Descriptif synthétique des missions effectuées

\begin{tabular}{|c|c|c|}
\hline Mission & Acteurs/Événements & Activités de recherche \\
\hline $\begin{array}{l}\text { 1' } \text { mission : } \\
\text { octobre } 2011\end{array}$ & $\begin{array}{l}\text { Séminaire annuel de I'IMV } \\
\text { Consultation des bibliothèques de } \\
\text { la ville: IMV, INSPC, IRD, Faculté } \\
\text { de géographie, de sciences } \\
\text { d'Antananarivo }\end{array}$ & $\begin{array}{l}\text { Recherche bibliographique et } \\
\text { prise de contact IMV et ONG EAST }\end{array}$ \\
\hline $\begin{array}{l}2^{\mathrm{e}} \text { mission : } \\
\text { janv-12 }\end{array}$ & $\begin{array}{l}\text { Rencontres différents acteurs : } \\
\text { Consolidation lien avec ONG EAST }\end{array}$ & $\begin{array}{l}\text { 1re phase d'enquêtes quantitative } \\
\text { et qualitative en matière } \\
\text { d'eau, d'assainissement et de } \\
\text { santé dans certains fokontany } \\
\text { d'Antananarivo. }\end{array}$ \\
\hline $\begin{array}{l}3^{\mathrm{e}} \text { mission : } \\
\text { mars-13 }\end{array}$ & $\begin{array}{l}\text { Rencontres différents acteurs: } \\
\text { - institutions: BDA } \\
\text { - académiques: } \\
\text { Professeur Ramamonjisoa } \\
\text { (géographe), Professeur } \\
\text { Louis Paul Randriamarolaza } \\
\text { (anthropologue), Professeur } \\
\text { Raymond (historien), } \\
\text { M. Rakotomalala } \\
\text { - ONG : Water Aid, GRETA }\end{array}$ & $\begin{array}{l}2^{\text {nde }} \text { phase d'enquêtes } \\
\text { quantitative et qualitative en } \\
\text { matière d'eau, d'assainissement } \\
\text { et de santé dans certains } \\
\text { fokontany d'Antananarivo. }\end{array}$ \\
\hline $\begin{array}{l}4^{\mathrm{e}} \text { mission : } \\
\text { mars-14 }\end{array}$ & $\begin{array}{l}\text { Colloque sur l'eau à la faculté des } \\
\text { sciences d'Antananarivo } \\
\text { Rencontre d'acteurs du secteur } \\
\text { WASH, Institut Pasteur }\end{array}$ & $\begin{array}{l}\text { Analyse de l'eau sur les bornes- } \\
\text { fontaines }\end{array}$ \\
\hline $\begin{array}{l}5^{\mathrm{e}} \text { mission : } \\
\text { juill-14 }\end{array}$ & $\begin{array}{l}\text { Rencontres avec } \\
\text { - institutions : coordonnateur } \\
\text { WASH, JIRAMA, ministère de la } \\
\text { santé, BMH } \\
\text { - ONG : ONU habitat, ENDA, OMS } \\
\text { - privé : SAMVA, APIPA, CITE }\end{array}$ & $\begin{array}{l}\text { Enquêtes auprès des médecins } \\
\text { chefs des CSB publics } \\
\text { d'Antananarivo }\end{array}$ \\
\hline
\end{tabular}

Tout d'abord, des enquêtes ménages sur les pratiques de l'eau ont été réalisées durant deux missions regroupant un échantillonnage de 300 personnes sur 12 fokontany (Ambohimandroso, ManjakarayIIB, Manjakaray IIC, Amboditsiry, Androhavangy tsena, Mahavoky, Antetezanafovoany I, Anatihazo Isotry, Mandrangobato I, Anosib Ambohibarikely, Ampamatanana, Ambohitsoa) (figure 1). D'après nos 50 premiers enquêtés, la grande majorité vit sous le seuil de pauvreté, c'est-à-dire avec moins d'un euro par jour. Pour les autres $(n=250)$, cette question concernant le niveau de vie a volontairement été omise tant elle constituait auprès des enquêtés un véritable frein à la poursuite de l'enquête et remettait ainsi en cause l'enquête elle-même. Les références aux origines n'ont pas été abordées. Cependant on note que seuls $34 \%$ des enquêtés déclarent vivre à Antananarivo depuis moins de dix ans. 
Puis, 22 entretiens ont été effectués auprès de focus group des professionnels de l'eau. Ces informateurs-clés constituent un maillon important de la chaîne de distribution de l'eau dans les pays africains car ils se trouvent au plus proche du quotidien des habitants (responsables, gérants de $\mathrm{BF}$, vendeurs ambulants). Parmi ses 22, 10 ont été réalisés auprès des participantes à une AFHy (Association féminine de promotion à l'hygiène) appartenant au réseau de l'ONG EAST. Cette association propose des actions journalières de sensibilisation à l'hygiène, des actions modèles de nettoyage et d'embellissement des quartiers (nettoyage de ruelles, ramassages et rejet des ordures, embellissement par plantations de fleurs, rôle de responsabilisation et d'insertion des femmes dans la vie des quartiers).

15 entretiens semi-directif focalisés sur les structures de santé et personnels de santé ont été menés (medecins-chefs des 12 CSB publics, 3 médecins du CHU, 1 du BMH, 3 médecins de quartiers, 1 sage-femme d'un CSB public). Ce réseau de personnes interrogées a servi à collecter des données quantitatives qui sont utilisées pour les analyses statistiques (ACP et CAH).

Enfin, 15 prélèvements à des fins d'analyse de potabilité complètent ce tableau : 10 aux bornes-fontaines, 1 dans une source, 4 auprès des usagers dont 2 dans des gargotes et 2 dans des ménages complètent ce tableau. Ces analyses ont été réalisées auprès du seul laboratoire autorisé à Antananarivo, l'Institut Pasteur. Deux séries de résultats d'analyse, commandées par l'entreprise de distribution de l'eau malgache la JIRAMA, apportent certains compléments. Même si, là encore, l'exploitation des données est limitée du fait de leurs natures. La première série effectuée au point de production et d'alimentation de l'eau potable n'identifie que les paramètres physiques de l'eau au niveau du lac (ressource brute), puis au niveau des sorties MI et MII (soit après traitement de l'usine). La seconde série aux bornes-fontaines ne présente qu'une vision incomplète puisque la pratique du tirage au sort ne permet pas une analyse sur le long terme (entre une dizaine et une trentaine de bornes-fontaines à chaque fois différente).

\section{III- Résultats de l'approche pluridimensionnelle : le statut ambivalent de la ressource en eau à Antananarivo à la fois menacée et menaçante}

Nous avons effectué une analyse du système d'approvisionnement en eau potable en ses 5 étapes selon le modèle précédemment défini. Notre diagnostic établit dans le contexte des quartiers précaires et denses de la capitale que la ressource en eau est à la fois menacée et menaçante tant en quantité qu'en qualité. 


\section{1- Une ressource menacée}

D'un point de vue quantitatif, nos résultats obtenus dans ces quartiers contrastent avec les données recueillies à l'échelle de la CUA par ARTELIA. Seuls $8 \%$ des ménages affirment être raccordés au réseau de distribution, loin des $33 \%$ annoncés par la Banque mondiale et des $53 \%$ par le SDAU (Banque mondiale, 2011 ; ARTELIA, 2014). La borne-fontaine apparaît dans les descriptions faites par les enquêtés comme un lieu public majeur dans la vie quotidienne des Antananariviens. Ainsi, les temps d'attente aux bornesfontaines peuvent être relativement longs, comme le symbolise la longue chaîne de bidons (supérieur à 30 minutes en moyenne et jusqu'à deux heures dans certains fokontany) (figure 4). Dès lors, les pratiques du transport et du stockage dominent (92\% des enquêtés). Le transport s'effectue essentiellement dans des jerricans ou des seaux d'eau. Comme dans la plupart des pays africains (Jaglin, 2005 ; Ginisty, 2014), la tâche de la collecte incombe le plus souvent à la femme et aux enfants, entrainant une organisation quotidienne et familiale spécifique (Ognard, 2018). Pour pallier le déficit de service public (Jaglin, 2005 ; Blanchon, 2006 ; Burt et Ray, 2014), des alternatives s'organisent autour de la revente d'eau. Les porteurs revendeurs d'eau ( « mpatsaka » rano) jouent un rôle important dans l'approvisionnement en eau, en particulier dans les quartiers difficiles d'accès. Ce service proposé aux alentours des bornes-fontaines s'adapte à une clientèle hétérogène composée de familles à revenu moyen ou faible. Ce service est en moyenne plus cher qu'à la borne-fontaine, le bidon de 20 litres étant acheté à $1 \mathrm{Ar} . / \mathrm{l}$ et revendu entre 50 Ar. (à Antetezana Afavoany). L'autre forme de revente d'eau est celle des particuliers. Si cette forme de revente peut être intégrée dans des stratégies familiales de solidarité, elle relève le plus souvent de stratégies marchandes comme à Mandrangobato où le prix pratiqué est sensiblement le même que celui appliqué aux bornes-fontaines (1 à 2 Ar. le litre). Dans l'ensemble, ces diverses stratégies attestent d'une part de la diversité des situations de mal branchement et de fait de l'adaptation urbaine avec un « artisanat de services informels » (Jaglin et Zérah, 2010). Le niveau de consommation d'eau est un autre indicateur des inégalités sociales par rapport à l'accès à l'eau. Le manuel des procédures du ministère malgache de l'énergie et des mines donne comme norme 30 litres/personne/jour quand la moyenne de l'OMS s'étend entre 20 et 50 litres/personne/jour. De nouveau, les résultats de nos enquêtes contrastent avec ceux d'ARTELIA où la consommation moyenne observée est de 32 litres/personne/jour. Selon nos enquêtes, $13 \%$ des ménages vivraient avec moins de 40 litres par jour par ménage, $38 \%$ avec 40 à 80 litres par jour par ménage, $15 \%$ avec 80 à 120 litres par ménage et $25 \%$ avec plus de 120 litres par ménage. Ces situations de mal branchement participent à des 
différenciations dans l'organisation quotidienne des familles contribuant ainsi à forger des modes de vie différenciés au sein de la capitale (Ognard, 2018).

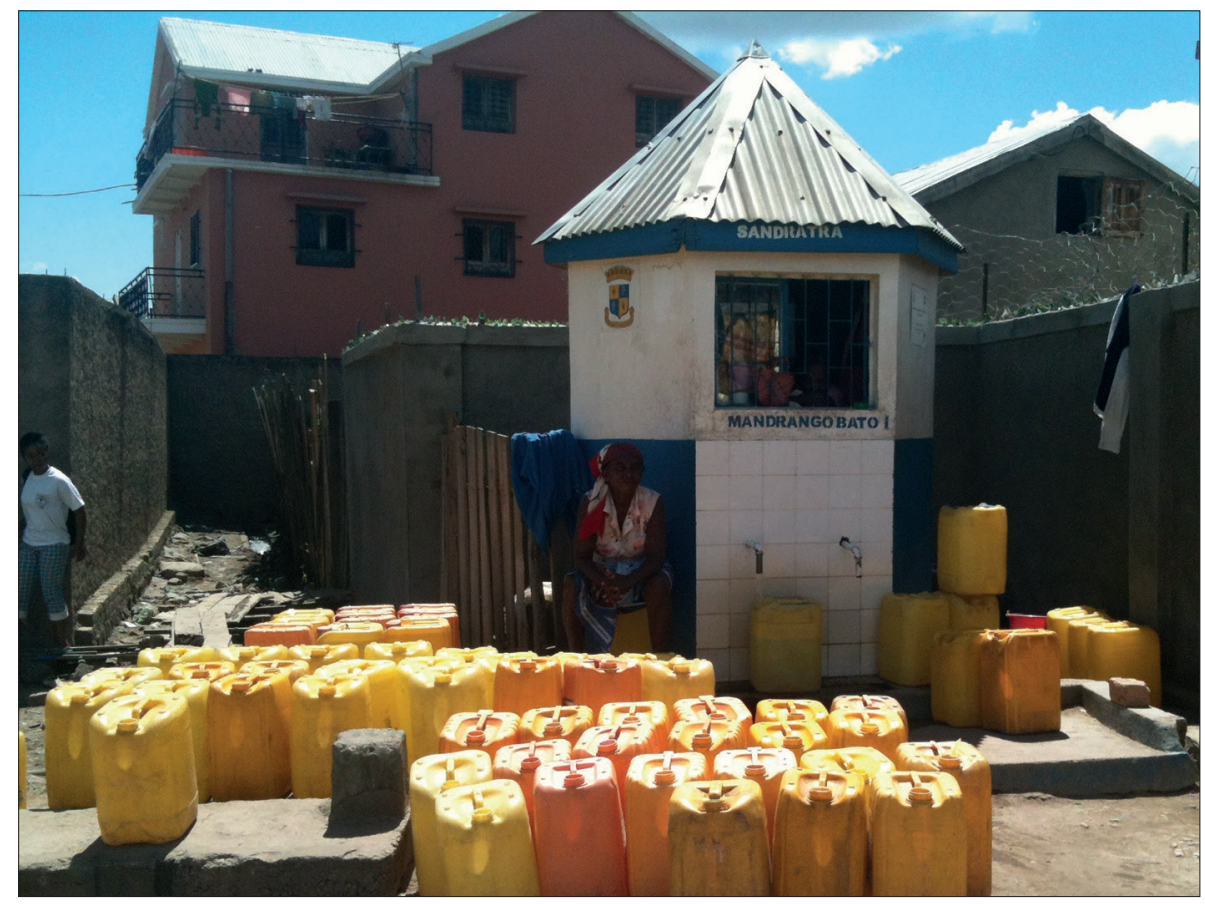

Figure 4 - Engorgement à la borne fontaine de Mandroganbato I Source : Ognard, 2018.

D'un point de vue qualitatif, les analyses microbiologiques de l'eau distribuée montrent une contamination d'origine fécale avec la présence de coliformes à une proportion qui dépasse la concentration maximale acceptable inférieure à un coliforme pour $100 \mathrm{ml}$ pour la consommation humaine sans risque sanitaire. Cette contamination a été notée en 7 sites sur les 15 prélevés avec une concentration d'escherichia coli variant de 8 à 13 coliformes/litres selon les lieux et une concentration de 25 à plus de 100 pour les entérocoques intestinaux. Ces 7 prélèvements non conformes montrent donc une contamination bactériologique significative de la qualité de l'eau consommée. La contamination à la fois par les germes escherichia coli et les entérocoques intestinaux définit des situations de péril fécal auxquelles sont soumises les populations.

Cependant, les paramètres physiques restent eux conformes à la réglementation. Ces données sont en partie conformes aux résultats des séries d'analyses de la JIRAMA. Au lieu de production, sur les 4 années (2009 à 2014) répertoriées seuls 3 évènements de pollution physique ont été identifiés. 
Ils ont eu lieu les 27/01/2009, 30/12/2009 et 05/03/2012. Le paramètre mis en cause est la turbidité qui supérieure à 5 NTU qui rend l'eau légèrement trouble. Le principal risque associé est alors l'augmentation de la capacité de transport de micro-organismes nuisant à la désinfection. À l'échelle des bornes-fontaines, pour les années 2013 et 2014, ce système de désinfection est incriminé. La turbidité est élevée sur 39 échantillons sur les 508 effectués sur les deux années. À noter que ce paramètre n'est pas pris en compte par la JIRAMA comme facteur de conformité de l'eau puisque ces échantillons ont été déclarés conformes à la norme, alors même que le seuil de 5 NTU, défini par la loi malgache et les recommandations de l'OMS, est ici dépassé. Cette dissension entre lieu de distribution et lieu de captage interroge le rôle joué par le transport et les canalisations.

Par ailleurs, les différents modes d'approvisionnement, c'est-à-dire la nature de la source, entrent également en ligne de compte pour l'appréciation du risque et du niveau de contamination de l'eau distribuée. La seule source analysée à Manjakaray semble contaminée par les entérocoques mettant en cause l'approvisionnement à une source non améliorée.

Deux prélèvements sur quatre effectués auprès des ménages présentent des contaminations d'origine bactériologiques. Là encore, la source du prélèvement est intéressante. Les deux prélèvements sains ont été effectués auprès de gargotes alors que ceux contaminés ont été effectués auprès des ménages. Le temps de stockage et le récipient peuvent être mis en cause. Les gargotes procèdent d'après nos enquêtes à un renouvellement régulier de leur approvisionnement en eau, ce qui n'est pas forcément le cas pour les ménages. D'autre part, l'absence d'altération de la qualité physique de l'eau permet d'émettre l'hypothèse que les manipulations et les conditions d'hygiènes sont ici en cause. Certains ont marqué une conscientisation de l'impact de leur manipulation sur la qualité de l'eau en affirmant l'utilisation d'un désinfectant, le Sur'Eau (14\% des enquêtés). Tsiny à Anatihazo déclare ainsi « l'eau est potable car j'utilise du Sur'Eau pour être sûr ». À l'inverse, à Anatihazo toujours, Jacqueline dénigre la qualité de l'eau en affirmant que l'eau n'est pas potable et qu'elle « doit rajouter du Sur'Eau ». Toutefois, de nombreux retours nous ont été faits sur la difficulté d'obtention de la solution de traitement en accord avec les observations des références bibliographiques (Dunston et al., 2001).

Compte tenu de la faible empreinte spatiale de nos analyses, cette expérience n'est pas représentative de l'ensemble de l'agglomération mais permet de dégager certaines tendances quant à la qualité de l'eau et aux pratiques des populations. 
Les perturbations qui détériorent l'accès en quantité et en qualité se concentrent autour des problématiques de transport : à l'échelle de la CUA, au niveau des canalisations mais également à l'échelle individuelle à travers les pratiques de transport et de stockage.

\section{2- L'eau, effluent, ressource menaçante}

Parallèlement, l'absence d'un système d'assainissement opérant amène une importante partie de la population à évacuer les eaux ménagères de manière inadéquate, favorisant un risque sanitaire (figure 5). $80 \%$ des personnes interrogées rejettent ainsi leurs eaux usées (cuisine, vaisselle, lessive) à l'air libre. Le raccordement au tout-à-l'égoût ne concerne qu'une minorité (7 \%). Cette donnée peut être complétée par l'équipement sanitaire : la fosse perdue (technique locale rudimentaire avec un simple trou) se présente comme le mode le plus répandu. Les latrines se trouvent le plus souvent à l'extérieur et sont partagées par plusieurs familles (72\% des réponses). On remarque que certaines pratiques comme les défécations «à l'air libre » peuvent augmenter le risque sanitaire. Le "péril fécal » reste une donnée sensible dans ces quartiers. Cette analyse peut être corroborée par les défaillances du système de récolte des ordures de la JIRAMA déjà mentionné dans l'ouvrage Antananarivo et ses ordures (Raharinjanahary, 2011) avec un manque de bacs, de personnels et de camions poubelles (478 bacs à ordures pour 1 million d'habitants). Les pratiques de la décharge sauvage comme l'attestent nombre de canaux obstrués de la ville ou encore les pratiques d'incinération et de mise au trou dans la cour $(23 \%)$ des déchets, ne font qu'accentuer le problème. En effet, l'Ikopa est le seul collecteur de tous les déchets liquides restitués à l'aval de la ville d'Antananarivo. Il reçoit sans aucun traitement les effluents urbains provenant de la station de pompage des 67 ha au niveau d'Antopenaka, les matières de vidanges transportées par camion au site d'Ambohitrimanjaka et les eaux polluées du canal Andriantany au niveau du village d'Andriantany (ARTELIA, 2014). La qualité environnementale et sanitaire de l'eau de ces effluents paraît compromise. Elle a été évaluée à l'échelle de la Commune Urbaine d'Antananarivo (CUA), grâce aux deux campagnes de prélèvements des eaux sur l'Ikopa effectuées en 2008 et 2013 (ARTELIA, 2014). Au cours de ces études, les usages de l'Ikopa (lessive, briques, sable, barrage) ainsi que les rejets d'effluents dans le cours d'eau (rejets municipaux et rejets industriels) ont été inventoriés, et une campagne de prélèvements d'échantillons et d'analyses d'eaux réalisée. Au travers de ces études sur les principales caractéristiques de la rivière Ikopa, la ressource apparaît de relative bonne qualité au niveau de la prise d'eau de Mandroseza, même si elle reçoit des effluents pollués en amont notamment industriels et domestiques (au niveau de la centrale thermique d'Ambohimanambola). 


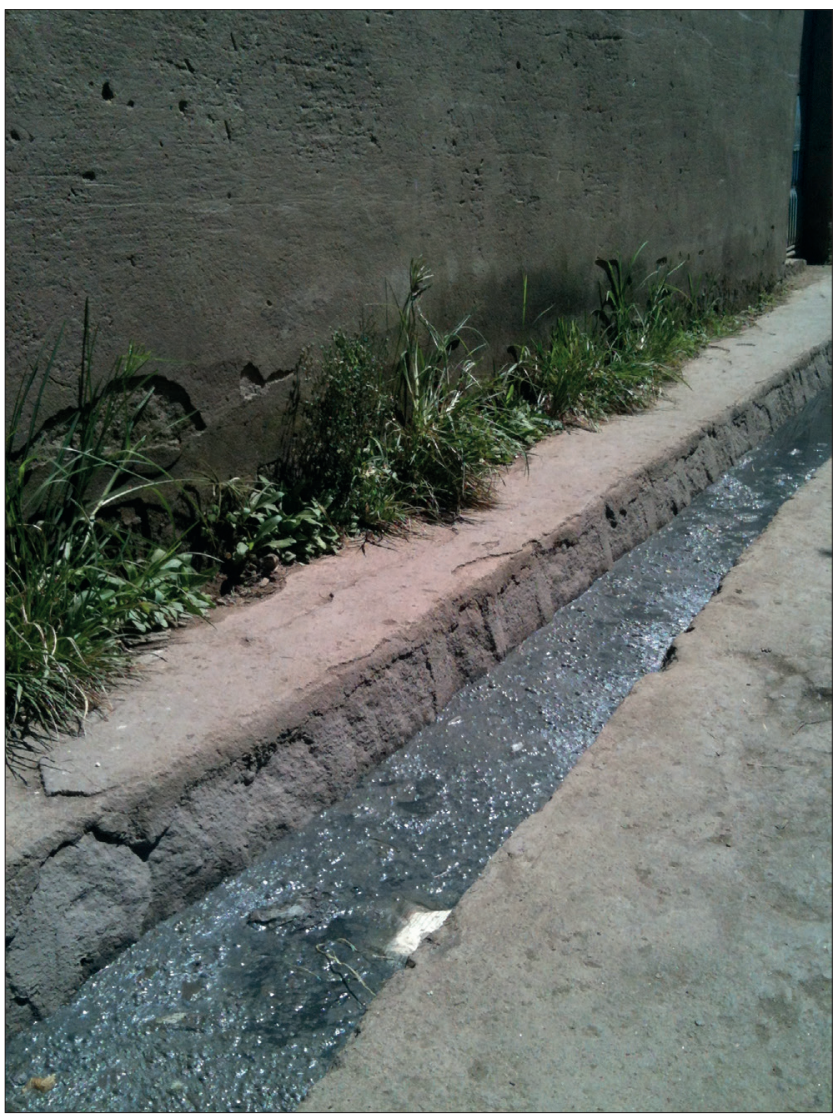

Figure 5 - Photographie de canal à ciel ouvert servant de réceptacle à ordures à Mandroganbato I

Source : Ognard, 2018.

L'analyse diachronique montre une dégradation de la qualité de la rivière Ikopa des 5 années entre 2008-2013. Elle est qualifiée de médiocre pour la plupart des sites de prélèvements avec des critères de $\mathrm{pH}$ inférieur à 5,5 et supérieur à 9,5, une conductivité comprise entre 500 et $3000 \mu \mathrm{S} / \mathrm{cm}$, une température comprise entre 30 et $35^{\circ} \mathrm{C}$. L'indicateur le plus parlant de cette dégradation de la qualité de l'Ikopa est la concentration en oxygène dissous du cours d'eau avec des niveaux compris entre 2 et $3 \mathrm{mg} / \mathrm{l}$. De plus, l'évolution de la consommation globale en eau affecte directement la quantité totale de production d'eaux usées. Avec une production d'eaux usées estimée à $80 \%$ des eaux consommées, la quantité totale d'eaux usées produite au niveau de la zone d'étude est de $1 \mathrm{~m}^{3} / \mathrm{s}$ en 2013. Si les objectifs d'amélioration de l'accès à l'eau potable sont respectés, cette production double en $2015(2 \mathrm{~m} / \mathrm{s})$ et atteindra $3,2 \mathrm{~m}^{3} / \mathrm{s}$ en 2025 . Comme la zone d'étude est drainée par le réseau 
hydrographique de la rivière Ikopa, la confrontation de ces flux avec les débits d'étiage de cette rivière, qui peuvent descendre à moins de $5 \mathrm{~m}^{3} / \mathrm{s}$ au niveau d'Ambohitrimanjaka, peuvent de plus en plus fragiliser la ressource et les quartiers en situation de la plaine inondable. En soulignant l'imbrication des réseaux d'assainissement à ceux de l'approvisionnement en eau, il ne s'agit pas seulement de questionner la mauvaise gestion des eaux usées (ARTELIA, 2014) mais d'identifier l'interdépendance de ces réseaux qui joue un rôle important dans la dégradation de l'environnement urbain. On parle de "vulnérabilité transversale » (Hardy, 2009). Elle est transcrite dans le profil épidémiologique de la capitale malgache. Selon les études épidémiologiques, l'incidence est le meilleur moyen d'appréhender l'évolution des maladies d'une population donnée (Vaughan et Morrow, 1991 ; OMS, 1994). Or, l'évaluation de cet indice n'est pas possible dans cette étude de cas étant donné la variabilité et le manque de fiabilité des données statistiques démographiques. L'étude s'appuie donc sur la prévalence, qui est le principal indicateur de la morbidité, s'appuyant sur le nombre total de maladies observées dans une population donnée à un moment précis. L'étude statistique ici présentée a été réalisée à partir des données de morbidité diagnostiquée par les Centres de Santé de Base (CSB) publics de la Commune urbaine d'Antananarivo (15 au total) dont la cohérence nous semble plus plausible que les données de mortalité fournies par le ministère. Dans un premier temps, à défaut de pouvoir évaluer l'incidence des maladies hydriques, une approche diachronique a été effectuée à partir des graphiques semi-logarithmiques pour établir l'évolution du phénomène morbide (figure 6). Les graphiques mettent en évidence des taux de croissance des maladies respiratoires et autres maladies qui semblent se comporter comme le taux de croissance total. Ainsi, l'existence de droites traduit des taux de croissance constants. De plus, les trois segments de droite sont proches et parallèles. Ceux-ci correspondent à des taux de croissance identiques. Les deux pathologies (maladies respiratoires et autres maladies) influencent donc fortement l'évolution de la distribution pathologique d'Antananarivo. Un deuxième groupe de pathologies se détache : les maladies diarrhéiques, des affections digestives et cutanées. Leurs taux de croissance présentent des profils sensiblement similaires avec des segments de droite qui connaissent de faibles variations de $10 \%$ à $-10 \%$. Ce deuxième groupe souligne donc la fragilité de la capitale malgache vis-à-vis des risques sanitaires hydriques notamment. Ces résultats amènent à dire, en se basant seulement sur la morbidité diagnostiquée, que l'état sanitaire, en identifiant le poids des maladies respiratoires et des maladies diarrhéiques, corrobore parfaitement une situation d'insalubrité et de précarité de nombreux quartiers de la ville d'Antananarivo. 

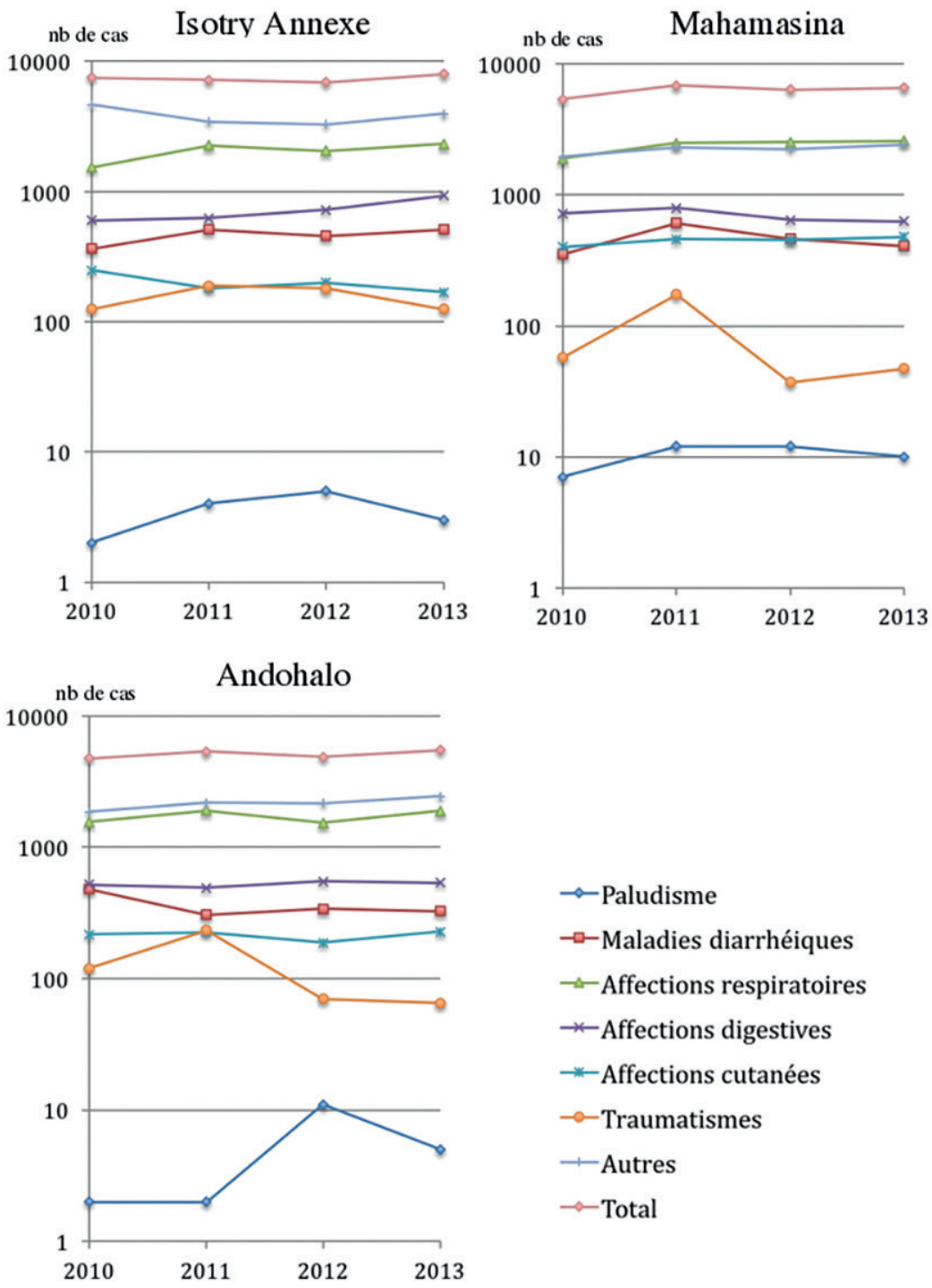

$$
\begin{aligned}
& \rightarrow-\text { Paludisme } \\
& \rightarrow \text {-Maladies diarrhéiques } \\
& \rightarrow-\text { Affections respiratoires } \\
& * \text { Affections digestives } \\
& \because \text { Affections cutanées } \\
& \rightarrow-\text { Traumatismes } \\
& \rightarrow \text { Autres } \\
& \rightarrow-\text { Total }
\end{aligned}
$$

Figure 6 - Évolution pathologique de 2010 à 2013 de 3 CSB publics

Source : CSB central d'Isotry, 2010-2013. Réalisation : Ognard, 2018.

Conjointement, une analyse en composante principale (ACP) et une classification ascendante hiérarchique $(\mathrm{CAH})$ ontété réalisées à partir des résultats de morbidité des $12 \mathrm{CSB}$ publics de la capitale (figures 7 et 8 ). Ces résultats transcrivent une partition géographique de l'espace urbain d'Antananarivo (figure 9). La Commune Urbaine d'Antananarivo apparaît scindée en deux, 
entre un Est aux conditions sanitaires relativement favorisées dans un contexte de ville de pays en développement (la variable « autres maladies » dominante) et un Ouest caractérisé par un profil moyen (où aucune maladie n'apparaît comme prépondérante). À l'intersection de ces deux ensembles, Isotry central, un quartier identifié comme précaire cristallise les pathologies caractéristiques des pays en développement : les maladies hygiéniques avec la prépondérance des maladies diarrhéiques, respiratoires et digestives.

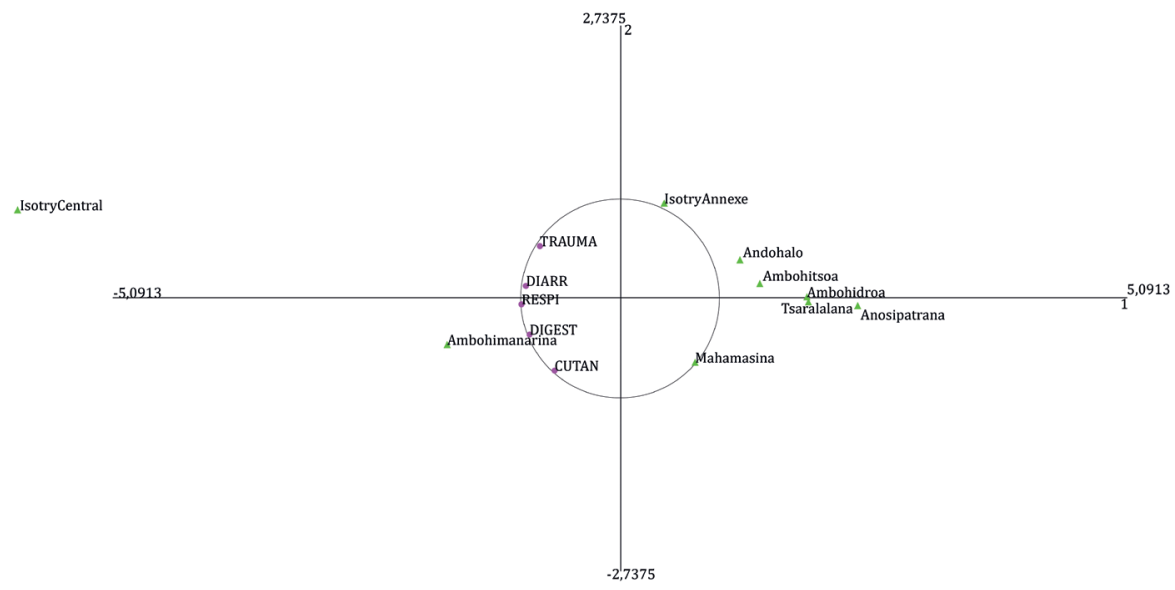

CSMIT

Figure $\mathbf{7}$ - Axes factoriels $\mathbf{1}$ et $\mathbf{2}$ de l'analyse en composante principale Source : CSB central d'Isotry, 2010-2013. Réalisation : Ognard, 2018.

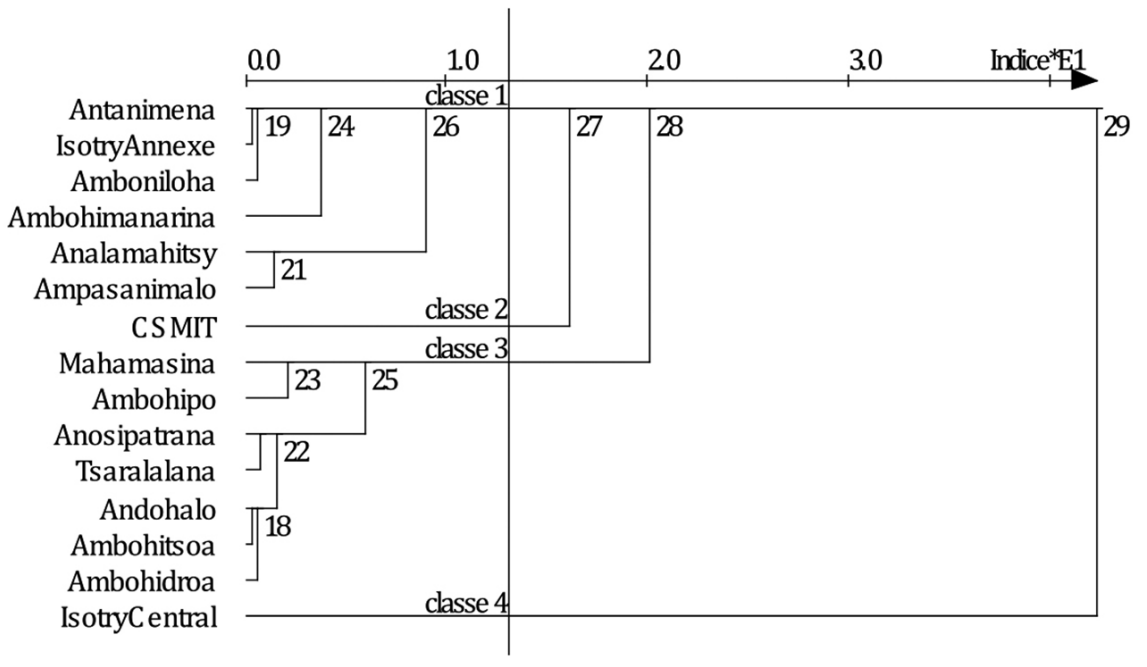

Figure 8 - Dendrogramme de la classification ascendante hiérarchique 


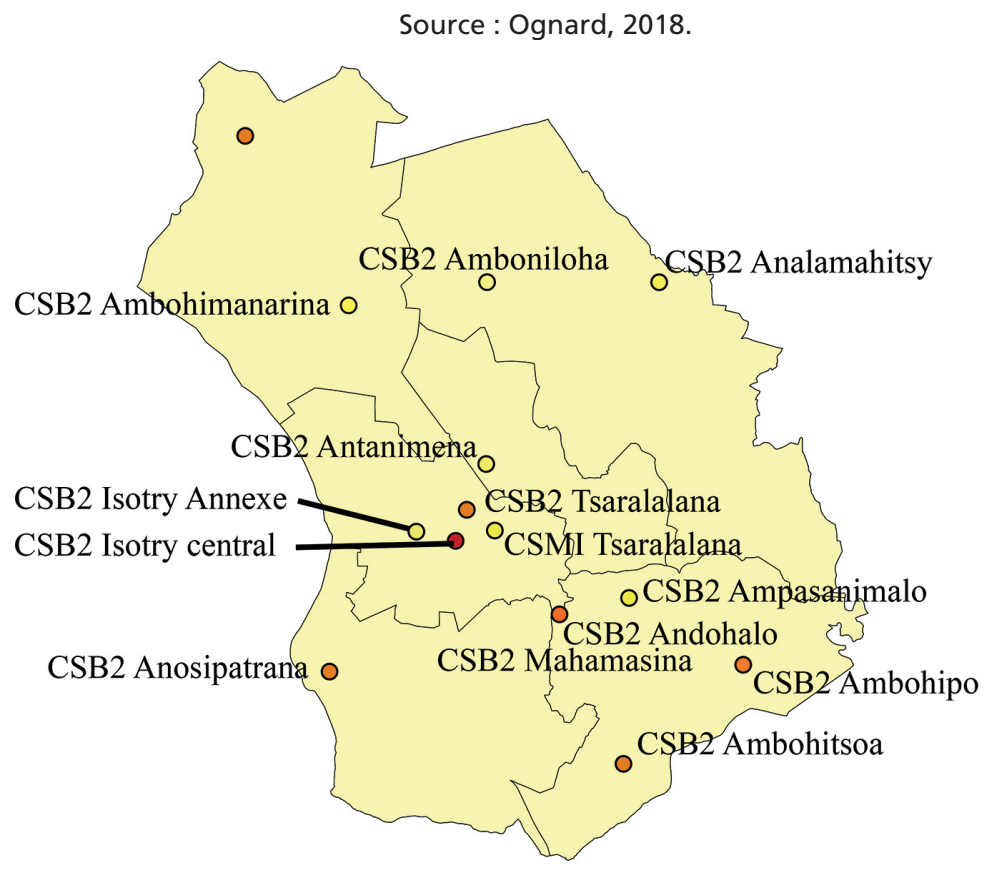

\section{Légende}

$0 \quad 2 \mathrm{~km}$

- CSB classe 1 : profil amélioré avec prépondérance de «autres maladies»

- CSB classe 2 : profil moyen avec aucune patholoie prépondérante

- CSB classe 3 : profil dégradé avec prépondérance des maladies diarrhéiques

Figure 9 - La partition géographique et sanitaire de la Commune urbaine d'Antananarivo selon la classification ascendante hiérarchique

Source : CSB central d'Isotry, 2010-2013. Réalisation : Ognard, 2018.

\section{IV- Discussion : dynamique du système d'eau antananarivien, de l'instrumentalisation à la résilience des quartiers précaires}

Cette double vulnérabilité linéaire et transversale de la ressource en eau de la capitale malgache atteste donc d'une fragmentation de l'espace urbain d'Antananarivo. Si Al Dabaghy (2014) a déjà noté le potentiel de « ressource politique transactionnelle de l'eau » à Diégo Suarez, cette double vulnérabilité de la ressource en eau dans la capitale malgache est en réalité le reflet de tensions entre au moins deux acteurs ; les élites et la population, en particulier 
les catégories les plus démunies. Elle pose la question de la gouvernance de la ressource en eau dans ce territoire.

\section{1- L'eau est à la fois " ressource " et risque : instrumentalisation et territorialisation de la ressource}

En effet, l'Ikopa est à la fois l'unique source d'eau potable de l'ensemble de l'agglomération d'Antananarivo, la principale source d'irrigation pour la plaine du Betsimitatatra et aussi le seul collecteur de toutes les nuisances restituées à l'aval de la ville d'Antananarivo. La préservation de la ressource en eau de l'Ikopa se retrouve compromise face à son statut ambivalent de source et d'effluent. En effet, si elle constitue la principale source d'alimentation de la capitale malgache, l'absence de traitement des eaux usées dans le petit cycle de l'eau est source de dangers, notamment sanitaires, pour l'ensemble de la CUA mais en particulier pour les quartiers précaires comme Isotry central. Ces risques sont particulièrement prégnants en saison pluvieuse et cyclonique où les bas-fonds intra-muros principalement situés à l'ouest de la capitale connaissent un débordement des canaux d'égouts à l'air libre. La ressource en eau devient un instrument de territorialisation de la capitale malgache où les services informels deviennent essentiels. En effet, l'absence de reconnaissance et d'encadrement de ces derniers est un autre exemple de cette volonté de cloisonnement de ces territoires et un attachement institutionnel à un modèle universel inadapté. Car cette inadaptation est le résultat d'un processus historique long de constitution des réseaux où les politiques successives ont oscillé entre efficacité et équité. Antananarivo n'a jamais véritablement connu l'âge d'or des réseaux de service public intégré et intégrateur au cours de laquelle aurait dû être réalisée leur universalisation. Dès 1945, une rupture est marquée avec l'équilibre écologique par l'occupation du lit majeur de l'Ikopa avec la création des quartiers d'Ampefiloha et d'Ambodin'isotry (ouest du lac Anosy) avec des fonctions désormais bien réparties entre ville « haute », devenue lieu de résidence des populations aisées, et « ville basse » devenant lieu du commerce, des loisirs et de l'habitat des populations les plus pauvres (Asinome, 2006). Cette marginalisation conduit à la fabrique de fractures sociales et urbaines qui sont entretenues dans le temps comme ici avec l'accès aux services publics. De même, au niveau sanitaire, la dichotomie du paysage urbain résultant des analyses statistiques correspond à celle opérée dans la mentalité malgache entre cet Est sur les hauteurs depuis longtemps mis en valeur par les Malgaches et cet Ouest caractérisé par cette situation de plaine inondable (derrière la digue) (Fournet-Guérin, 2006). On peut donc dire ici que le « territoire de santé » semble fortement imprégné des représentations et donc du « territoire vécu » (Fleuret, 2015). Pourtant, si le profil sanitaire 
dégagé pour l'Est confirme son statut privilégié au sein de la commune urbaine, le profil moyen dégagé pour l'Ouest nuance et atténue la stigmatisation de cette partie du territoire. Seul Isotry central s'impose par cette cartographie comme un «bas quartier» au sens où l'entend l'imaginaire malgache (autant socialement que géographiquement). Cependant, le CSB Isotry central joue un rôle particulier dans la carte sanitaire. Il coordonne et centralise l'ensemble des CSB de la commune urbaine. Ce quartier présente à la fois une meilleure offre de soin et également une position d'accessibilité. Ceci pourrait donc expliquer son poids prépondérant.

\section{2- Les populations, à la fois victimes et actrices}

À l'échelle des individus, l'altération de la qualité de l'eau produite par les manipulations dans la sphère domestique engendre des risques sanitaires pour des utilisateurs qui sont à la fois victimes et acteurs. Ils sont victimes d'un système déficient avec un accès faible en quantité. Mais ces populations marginalisées ont réussi à mettre en place des stratégies avec l'instauration d'autres choix pour pallier les services publics. Les actions privées apparaissent comme prépondérantes et peuvent être intégrées comme des « coping strategies ». Ces stratégies rendent ainsi la ville « habitable » pour les citadins les plus pauvres (Jaglin, 2005). Se déplacer pour aller chercher l'eau, la puiser, la transporter dans des récipients en plastique posés sur la tête ou portés à bout de bras sont des actes qui se répètent plusieurs fois par jour. C'est alors à la notion de résilience et notamment à la capacité de réponses de l'individu que l'on s'intéresse. Cette dimension est intéressante car en se plaçant à l'échelle des individus, on présuppose qu'ils ont une certaine latitude dans leurs choix et intègrent les aléas dans leurs arbitrages. Les stratégies adoptées par les agents dépendent de la perception qu'ils ont des risques auxquels ils font face et des ressources qu'ils sont en mesure de mobiliser. La prise de décisions s'accompagne d'un arbitrage dans les ressources entre le court et le moyen terme selon le risque perçu et subi. Ces décisions sont prises dans un contexte d'incertitudes selon les informations dont ils disposent. Ici, les menaces naturelles ne sont pas seulement perçues et vécues d'après la représentation de leurs effets, elles entrent en concurrence avec d'autres contraintes d'ordre économique et social qui s'en trouvent relativisées. Toutes ces stratégies attestent que les risques urbains ou sociaux (pauvreté, mal logement) apparaissent plus prégnants que les risques naturels (inondations) et sanitaires (risques de maladies hydriques et d'hygiène). Les enjeux autour des valeurs, des types d'individus, des types familiaux se trouvent dès lors posés dans l'appréciation de la perception des risques et donc d'une multitude d'inégalités face aux risques. À Antananarivo, en particulier dans les quartiers 
enquêtés en situation de plaine, les populations ont la conscience du risque, mais une sorte de résignation est entérinée faute de moyens. Ainsi, un couple à Isotry expliquait qu'il déposait ses déchets au pas de leur porte ou à proximité dans le quartier car il «n'a pas d'autre choix ». Il est conscient que cette pratique entrave potentiellement sa santé, notamment au moment de la montée des eaux lors des inondations. On assiste à une acceptation du risque et au développement de pratiques originales qui participent de la fabrication urbaine, de « bricolages urbains ».

\section{Conclusion}

L'analyse du système d'eau potable en ces 5 étapes a permis d'identifier une double vulnérabilité de l'approvisionnement de la ressource en eau, à la fois « linéaire » et "transversale » dans la capitale malgache. Le système d'eau potable salubre interroge directement le rôle du transport, non seulement au niveau des canalisations mais surtout à travers les pratiques de revente et les manipulations domestiques puisque l'approvisionnement des populations se fait essentiellement aux bornes-fontaines. Si ces « coping strategies » permettent l'intégration des populations les plus pauvres dans la ville légale, ils mettent en évidence la fragmentation socio-spatiale de la capitale malgache. Parallèlement, l'attachement institutionnel à un modèle universel inadapté avec la persistance d'un statut ambivalent de l'Ikopa à la fois source et effluent, participe à la fabrique du risque et à un laisser-faire par les autorités du cloisonnement des territoires les plus fragiles de la capitale. Ce bien vital qu'est la ressource en eau est ainsi instrumentalisé et territorialisé.

\section{Bibliographie}

Al Dabaghy C., 2014 - « Le Prix de l'eau, hiérarchies urbaines, voisinage hydrique et communalité à Diego-Suarez ». Études rurales, n 194, p. 272.

Amrose S., Burt Z., Ray I., 2015 - « Safe drinking water for low-income regions », Annual review of environment and ressources, vol. 40, pp. 203-231.

ARTELIA, 2014 - Élaboration du schéma directeur de l'assainissement urbain du Grand Tana. Antananarivo, 202 p.

Asinome E., 2006 - S'approvisionner en eau potable dans les villes du Sud. Les quartiers défavorisés d'Antananarivo, Madagascar. Antananarivo, Université d'Antananarivo p. 8.

Aubriot O., Riaux J., 2013 - « Savoirs sur l'eau : les techniques à l'appui des relations de pouvoir? », Autrepart, ${ }^{\circ}$ 65, pp. 3-26.

Banque Mondiale., 2011 - Rapport "L'urbanisation ou le nouveau défi malgache". Washington. Institut for local resilience, $135 \mathrm{p}$. 
Bakker K., 2003 - « Archipelagos and networks : Urbanisation and water privatization in South ». The Geographical Journal, vol. 169, n 4, pp. 328-341.

Bayat A., 2000 - «From "dangerous classes" to "quiet rebels": The politics of the urban subaltern in the global South ». International Sociology, vol. 15, $\mathrm{n}^{\circ} 3$, pp. 533-557.

Blanchon D., 2010 - L'eau, une ressource menacée ? Paris, La Documentation photographique, $\mathrm{n}^{\circ} 8078,64 \mathrm{p}$.

Bravard J.-P., Honegger A., 2006 - « La pénurie d'eau : donnée naturelle ou question sociale ? » Géocarrefour, vol. 81, $\mathrm{n}^{\circ} 1$, pp. 3-4.

Bromley R., 1978 - « The urban informal sector: Why is it worth discussing? » World Development, vol. 6, n 9, pp. 1033-1039.

Burt Z., Ray I., 2014 - « Storage and non-payment: Persistent informalities within the formal watersupply of Hubli-Dharwad, India ». Walter Alternatives, $\mathrm{n}^{\circ} 7$, pp. 106-120.

Casciarri B., Van Aken M., 2013 - « Anthropologie et eau(x) affaires globales, eaux locales et flux de cultures », Journal des anthropologues, pp. 132-133.

Chardon K., 1996 - «Alimentation en eau d'une capitale d'Afrique tropicale, Antananarivo, Madagascar, Implication socio-économique du développement du réseau d'eau ». Géo regards, n 41, pp. 1-124.

CUA., 2014 - Plan multi-risques de préparation et de réponses aux catastrophes. Antananarivo, $80 \mathrm{p}$.

D’Ercole R., Metzger P. 2009 - « La vulnérabilité territoriale : une nouvelle approche des risques en milieu urbain ». Cybergeo : European Journal of Geography, n 447.

De Soto. H., 2000 - The mystery of capital. Why capitalism triumphs in the West and fails everywhere else. New York, Basic Books, 304 p.

Dos Santos S., 2005 - «Koom La Viim' : enjeux socio-sanitaires de la quête de l'eau à Ouagadougou ». Thèse de démographie. Université de Montréal, $182 \mathrm{p}$.

Dunston C., McAfee D., Kaiser R., Rakotoarison D., Rambeloson L., Quick R., 2001 - « Collaboration, cholera, and cyclones: a project to improve point-of-use water in quality in Madagascar ». American journal of public health, vol. 91, n 10, pp. 1574-1676.

Falkenmark M., 1989 - « The massive water scarcity threatening AfricaWhy isn't it being addressed ». Ambio, ${ }^{\circ} 13$, pp. 112-118.

Fleuret S., 2015 - «Construction locale de la santé : quels ont les facteurs de réussite des projets locaux de santé communautaire ». L'espace politique, $n^{\circ} 26$.

Fournet-Guérin C., 2006 - « Vivre le quartier à Tananarive. De la remise en cause d'un mythe urbain universel », Espaces et sociétés, n ${ }^{\circ} 126$, p. 69-86 
Fremigacci J., 2014 - État, économie et société coloniale à Madagascar. Paris. Khartala, 616 p.

Ginisty K., 2014 - Inégalités et (in)justices spatiales à Maputo : pratiques des services urbains. Thèse de géographie. Université Paris 10.

Hardy S., 2009 - «La vulnérabilité de l'approvisionnement en eau dans l'agglomération pacénienne : le cas du sous-système El Alto ». Cybergéo : European Journal of Geography, $\mathrm{n}^{\circ} 457$.

Jaglin S., 2005 - Services d'eau en Afrique subsaharienne : la fragmentation urbaine en question. Paris, CNRS Éditions, 244 p.

Jaglin S., Zérah M-H., 2010 - « Eau des villes : repenser des services en mutation. Introduction ». Revue Tiers Monde, $\mathrm{n}^{\circ}$ 203, pp. 7-22.

Jemmali H., 2013 - « Mesures de la pauvreté en eau : analyse comparative et développement de l'indice de pauvreté en eau ». Vertigo, $\mathrm{n}^{\circ} 2$.

Lavie E., Maupin A. et Blanchon D. 2015 - «La pénurie de l'eau, un discours à quelles fins ? » in Redon M., Magrin G., Chauvin E., Perrier Bruslé L. et E. Lavie (dir.). Ressources mondialisées. Essais de géographie politique. Paris, Publications de la Sorbonne, coll. « Territoires en mouvements », pp. 154-183.

Lavie E., Hassan El-Tayib N., 2014 - « Du robinet au consommateur : qualité de l'eau potable dans le contexte domestique de l'agglomération de Khartoum, Soudan ». Cybergéo, $\mathrm{n}^{\circ} 664$.

Ministère de la santé publique, 2007-2012 - Annuaires statistiques du secteur santé de Madagascar. Antananarivo.

N'Guimalet C.R., Balikouzou-Hinna D.A., Rasoanantoando GothardBassebe M.C., Semballa S., 2005 - «Gestion de la qualité de l'eau, conflits et risques dans la ville de Bangui (République centrafricaine) », Géocarrefour, vol. 80, n 4, URL : http://geocarrefour.revues.org/1295. Consulté le 26 mai 2011.

Ognard C., 2018 - Approche de la complexité des risques sanitaires et hydriques dans les quartiers précaires d'Antananarivo: la notion de contexte à l'épreuve des pratiques et représentations citadines. Thèse de géographie. Université de la Réunion, 329 p.

OMS, JMP, 2014 - Progress on Drinking Water and Sanitation: 2014 Update. New York: WHO/JMP.

OMS, 2014 - Preventing Diarrhea through Better Water Sanitation and Hygiene : Exposures and Impacts in Low- and Middle-Income Countries, Genève, Suisse, WHO.

Onda K., Lobuglio J., Batram J., 2012 - « Global acces to safe water: accounting for water quality and resulting impact on MDG progress », Journal of Environnemental Research and Public health, vol. 9, n 3, p. 880-894.

ONU, 2010 - Resolution on Human Rights and Acess to Safe Drinking Water and Sanitation. New York : UN. 
Reghezza-Zitt M., Ruffat S., 2015 - Resiliences. Sociétés et territoires face à l'incertitude, Aux risques et aux catastrophes. Londres, ISTE Éditions, $226 \mathrm{p}$.

Ruffat S., 2015 - Critique de la resilience pure in Reghezza-Zitt M. et Ruffat S. Resiliences. Sociétés et territoires face à l'incertitude, Aux risques et aux catastrophes. Londres, ISTE Editions, $226 \mathrm{p}$.

Rusca M., Boakye-Ansah A., Loftus A., Ferrero G., Van Der Zaag P., 2017 - « An interdisciplinary political ecology of drinking water quality. Exploring socio-ecological inequalities in Lilongwe's water supply network », Geoforum, vol. 84, pp. 138-146.

Raharinjanahary R., 2011 - Antananarivo et ses ordures, production, collecte, valorisation. Foi, justice éditions. Antananarivo, $269 \mathrm{p}$.

Sy I., Moussa K., 2014 - «Eau, hygiène, assainissement et santé dans les quartiers précaires de Nouakchott (Mauritanie) : contribution à l'approche écosanté à Hay Saken ». Vertigo, n 19.

Taglioni F., 2019 - «Zika et autres arboviroses » in Fleuret S., GasquetBlanchard C. et Hoyez A-C. (dir.), Abécédaire de la géographie de la santé, Paris, Éditions Matériologiques, pp. 219-227. 